University of Texas at El Paso

ScholarWorks@UTEP

$12-2010$

\title{
Fusing Continuous and Discrete Data, on the Example of Merging Seismic and Gravity Models in Geophysics
}

Omar Ochoa

The University of Texas at El Paso, omar@miners.utep.edu

Aaron Velasco

The University of Texas at El Paso, velasco@geo.utep.edu

Vladik Kreinovich

The University of Texas at El Paso, vladik@utep.edu

Follow this and additional works at: https://scholarworks.utep.edu/cs_techrep

Part of the Computer Engineering Commons

Comments:

Technical Report: UTEP-CS-10-51a

Published in Proceedings of the 30th Annual Conference of the North American Fuzzy

Information Processing Society NAFIPS'2011, El Paso, Texas, March 18-20, 2011.

\section{Recommended Citation}

Ochoa, Omar; Velasco, Aaron; and Kreinovich, Vladik, "Fusing Continuous and Discrete Data, on the Example of Merging Seismic and Gravity Models in Geophysics" (2010). Departmental Technical Reports (CS). 656.

https://scholarworks.utep.edu/cs_techrep/656

This Article is brought to you for free and open access by the Computer Science at ScholarWorks@UTEP. It has been accepted for inclusion in Departmental Technical Reports (CS) by an authorized administrator of ScholarWorks@UTEP.For more information, please contact Iweber@utep.edu. 


\title{
Fusing Continuous and Discrete Data, on the Example of Merging Seismic and Gravity Models in Geophysics
}

\author{
Omar Ochoa $^{1,2}$, Aaron Velasco ${ }^{1,3}$, Vladik Kreinovich ${ }^{1,2}$ \\ ${ }^{1}$ Cyber-ShARE Center of Excellence: A Center for Sharing \\ Cyberresources to Advance Science and Education \\ Departments of ${ }^{2}$ Computer Science and ${ }^{3}$ Geological Sciences \\ University of Texas at El Paso, El Paso, TX 79968, USA \\ omar@miners.utep.edu,velasco@geo.utep.edu,vladik@utep.edu
}

\begin{abstract}
In many application areas, we need to fuse continuous and discrete models of the same phenomena. For example, in geophysics, we have two main models for describing how the sound velocity changes with location and depth: a discrete gravity-based model, in which we have several layers with abrupt transition between layers, and a seismic model, in which the velocity continuously changes with the change in location and depth - and a transition is represented by a steeper change. Due to inevitable uncertainty, in two fused models, the same actual transition is placed at slightly different depths.

If we simply fuse these models, the fused model will inherit both nearby transitions and therefore, will, misleadingly, correspond to two nearby transitions instead of one. It is therefore necessary, before fusing, to first get a fused (more accurate) location of the transition surface.
\end{abstract}

In this paper, we show how to find such a location.

\section{Formulation of the Problem}

Need for model fusion. In many application areas, there are several different sources of data.

For example, in geophysics, one of the main problems is determining the density $\rho$ at different locations and different depths. It is known that for most minerals, density is uniquely related to the speed of sound, so determining density $\rho$ at different locations and depths is equivalent to determining the sound velocity $v$ at different locations and depths.

In geophysics, the two main sources of data for determining the density $\rho$ (or, equivalently, the sound velocity $v$ ) are:

- the seismic data, i.e., the arrival times of signal from earthquake (passive seismic data) and from the experimental explosions (active seismic data); and

- the gravity data, i.e., the values of the gravitational force at different locations.

Both data provide complementary information about the density:

- seismic data provides information about a narrow zone around a path from the source to the sensor, enabling us to determine density around this path with a high spatial resolution; on the other hand, seismic data only covers a direct vicinity of the paths, so it leaves many areas barely covered
- on the other hand, the gravity value at a given location is affected by the densities in the wide area around this location; thus, the gravity data provides information about the larger area - but with much smaller spatial resolution.

It is therefore desirable to use both types of data when estimating the values of density at different locations and lengths - what geophysicists call a density model; see, e.g., [1], [2], [3], [6], [7].

At present, there are no efficient algorithms for processing both types of data. So, to use both types of models, we must fuse the results of processing these two types of data: a seismic model that is obtained by processing seismic data, and a gravity model that is obtained by processing gravity data; algorithms for model fusions are described in [9], [10].

Computational problem: need to fuse discrete and continuous models. Traditionally, seismic models are continuous in the sense that in these models, the velocity smoothly changes as we change the location and/or depth. In contrast, the gravity models are discrete: in these models, we have layers, in each of which the velocity is constant, with an abrupt transition between layers.

The abrupt transition corresponds to a steep change in the continuous model. The problem is that both models describe the location of the transition only approximately, the corresponding transitions are located at slightly different depths. So, if we simply combine the corresponding values value-byvalue, e.g., by taking a weighted average of values corresponding to different locations and depths, then the resulting fused model will have two different abrupt transitions instead of one:

- one transition where the continuous model has it, and

- another transition nearby where the discrete model has it.

What we plan to do. To avoid the misleading doubletransition models, it is desirable, before fusing the models, to first fuse the corresponding transition locations. In this paper, we provide an algorithm for such location fusion.

Specifically, first, we formulate the problem in the probabilistic terms (see, e.g., [12]), and provide an algorithm that 
solves the corresponding probabilistic problem and produces the most probable transition location. Then, we show that the result of the probabilistic location algorithm is in good accordance with common sense - which reassures us that this location is reasonable. We also show how the commonsense intuition can be reformulated in fuzzy terms (see, e.g., [5], [8]).

\section{Available Data: What is Known And What NeEds to Be Determined}

For each location, in the discrete model, we have the exact depth $z_{d}$ at which we have a transition between the two layers. In contrast, for the continuous model, we do not have the abrupt transition; instead, we have velocity values $v(z)$ at different depths. We must therefore extract the corresponding transition value $z_{c}$ from the velocity values.

To be more precise, we have values $v_{1}, v_{2}, \ldots, v_{i}, \ldots, v_{n}$ corresponding to different depths. We need to find $i$ for which the transition occurs between the depths $i$ and $i+1$.

\section{Probabilistic APPROACH}

Description of the model. It is reasonable to assume that, with the exception of the transition point, for all other values $j$, the difference $\Delta v_{j} \stackrel{\text { def }}{=} v_{j}-v_{j+1}$ is small. This difference is caused by many different factors, so it is reasonable to invoke the Central Limit Theorem and assume that this difference is normally distributed with 0 mean and some standard deviation $\sigma$; see, e.g., [12]. The corresponding probability density is equal to

$$
p_{j} \stackrel{\text { def }}{=} \frac{1}{\sqrt{2 \cdot \pi} \cdot \sigma} \cdot \exp \left(-\frac{1}{2 \cdot \sigma^{2}} \cdot\left(\Delta v_{j}\right)^{2}\right) .
$$

We assume that differences corresponding to different depths $j$ are independent.

The value $\Delta v_{i}$ at the transition depth $i$ is not described by the normal distribution, it has to be given separately.

The resulting model is described by three parameters:

- the standard deviation $\sigma$,

- the transition depth $i$, and

- the transition values $\Delta v_{i}$.

Due to independence of different depth, the overall likelihood $L_{i}$ of the model with given values of these parameters is determined by the formula

$$
L_{i}=\prod_{j \neq i} p_{j}=\prod_{j \neq i} \frac{1}{\sqrt{2 \cdot \pi} \cdot \sigma} \cdot \exp \left(-\frac{1}{2 \cdot \sigma^{2}} \cdot\left(\Delta v_{j}\right)^{2}\right) .
$$

How to find the location: the general idea of the Maximum Likelihood Approach. In the probabilistic approach, we usually select the parameters for which the likelihood of the observed data is the largest; see, e.g., [12]. In other words, in this Maximum Likelihood Approach, we select the values of the parameters for which the likelihood $L$ attains the largest possible value.

How to find the optimal location $i_{0}$ : analysis of the corresponding optimization problem. Due to the fact that $\exp (a) \cdot \exp (b)=\exp (a+b)$, the expression $L_{i}$ can be represented as

$$
L_{i}=\frac{1}{(\sqrt{2 \cdot \pi} \cdot \sigma)^{n-2}} \cdot \exp \left(-\frac{1}{2 \cdot \sigma^{2}} \cdot \sum_{j \neq i}\left(\Delta v_{j}\right)^{2}\right) .
$$

The factor in front of the exponent does not depend on the location $i$ at all, so $L_{i}$ is the largest if and only if the exponential term is the largest:

$$
\exp \left(-\frac{1}{2 \cdot \sigma^{2}} \cdot \sum_{j \neq i}\left(\Delta v_{j}\right)^{2}\right) \rightarrow \max _{i}
$$

The function $\exp (-z)$ is strictly decreasing, so it attains its largest possible values when $z$ is the smallest. Thus, to find the optimal location $i$, we must find the value $i$ for which the following expression is the smallest:

$$
\frac{1}{2 \cdot \sigma^{2}} \cdot \sum_{j \neq i}\left(\Delta v_{j}\right)^{2} \rightarrow \min _{i} .
$$

Again, the factor in front of the sum does not depend on $i$, so this expression is the smallest if and only the sum attains its smallest value:

$$
\sum_{j \neq i}\left(\Delta v_{j}\right)^{2} \rightarrow \min _{i}
$$

This sum can be represented as

$$
\sum_{j \neq i}\left(\Delta v_{j}\right)^{2}=\sum_{j=1}^{n-1}\left(\Delta v_{j}\right)^{2}-\left(\Delta v_{i}\right)^{2} .
$$

The first term in this expression does not depend on $i$ at all. Thus, the above difference is the smallest if and only if the value $\left(\Delta v_{i}\right)^{2}$ is the largest. This, in turn, is equivalent to $\left|\Delta v_{i}\right|$ being the largest.

Thus, we arrive at the following conclusion.

Resulting location. As the most probable location of the transition point, we select the depth $i_{0}$ for which the absolute value $\left|\Delta v_{i}\right|$ of the difference $\Delta v_{i}=v_{i+1}-v_{i}$ is the largest possible.

Comment. This conclusion seems to be very reasonable: the most probable location of the actual abrupt transition between the layers is the depth at which the measured difference is the largest.

\section{The Results of the Probabilistic Approach are In GoOd ACCORDANCE WITH COMMON SENSE}

Common sense description of the problem. Intuitively, for each depth $i$, our confidence that this is a transition point depends on the actual value of the corresponding difference $\left|\Delta v_{i}\right|$ :

- the smaller the difference, the less confident we are that this is the actual transition depth, and

- the larger the difference, the more confident we are that this is the actual transition depth. 
The results of the probabilistic model are in accordance with common sense. Thus, the fact that in our probabilistic model, we select a location with the largest possible value $\left|\Delta v_{i}\right|$ show that the probabilistic model is in good accordance with common sense - which this increases our confidence in this result.

It may be useful to formulate the common sense description in fuzzy terms. Fuzzy logic (see, e.g., [5], [8]) is known to be a useful way to formalize imprecise commonsense reasoning. To apply fuzzy logic to our situation, we need to determine the degree of confidence $d_{i}$ that the transition occurs at the depth $i$. The above commonsense idea means that this degree of confidence $d_{i}$ is equal to $f\left(\left|\Delta v_{i}\right|\right)$, for some monotonically increasing function $f(z)$.

If we need to select a single location $i_{0}$, it is reasonable to select a value for which our degree of confidence is the largest

$$
d_{i}=f\left(\left|\Delta v_{i}\right|\right) \rightarrow \max .
$$

Since the function $f(z)$ is strictly increasing, this function attains its largest possible values when $z$ is the largest. Thus, to find the optimal location $i_{0}$, we must find the value $i$ for which the expression $\left|\Delta v_{i}\right|$ is the largest possible.

Of course, to come up with this conclusion, we do not need to use fuzzy logic, this conclusion can already be deduced from the above commonsense description. However, this description may be useful if we have other expert information that we need to combine with this one.

\section{How Accurate Is This Location Estimate?}

Formulation of the problem. The location $i$ that we obtained is approximate. How accurate is this location estimate?

Which approach should we use to solve this problem? We know that - at least for location - both models lead to the same result. It is therefore reasonable to select one of the models.

Since probability theory has been developed for centuries, so more methods and techniques have been developed - we will use the probabilistic approach.

Auxiliary result: estimating $\sigma$. In the probabilistic model, in addition to the location $i$, we also need to select the standard deviation $\sigma$.

We have already shown that the value $i$ can be determined by the Maximum Likelihood method. A similar Maximum Likelihood approach can be used to determine $\sigma$. Specifically, we can find $\sigma$ for which the expression

$$
L_{i}=\frac{1}{(\sqrt{2 \cdot \pi} \cdot \sigma)^{n-2}} \cdot \exp \left(-\frac{1}{2 \cdot \sigma^{2}} \cdot \sum_{j \neq i}\left(\Delta v_{j}\right)^{2}\right)
$$

takes the largest possible value over all possible $i$ and $\sigma$. We already know that with respect to $i$, the largest value is attained when $i$ is equal to the above estimate $i_{0}$, so we can simply substitute $i_{0}$ into the above expression:

$$
L_{i_{0}}=\frac{1}{(\sqrt{2 \cdot \pi} \cdot \sigma)^{n-2}} \cdot \exp \left(-\frac{1}{2 \cdot \sigma^{2}} \cdot \sum_{j \neq i_{0}}\left(\Delta v_{j}\right)^{2}\right)
$$

and maximize the result over $\sigma$.

Since the function $-\ln (z)$ is strictly increasing, this is equivalent to finding $\sigma$ for which the value $\psi \stackrel{\text { def }}{=}-\ln \left(L_{i_{0}}\right)$ is the smallest possible. This value has the form

$$
\begin{gathered}
\psi=(n-2) \cdot \ln (2 \cdot \pi)+(n-2) \cdot \ln (\sigma)+ \\
\frac{1}{2 \cdot \sigma^{2}} \cdot \sum_{j \neq i_{0}}\left(\Delta v_{j}\right)^{2} .
\end{gathered}
$$

Differentiating this expression by $\sigma$ and equating the derivative to 0 , we conclude that

$$
(n-2) \cdot \frac{1}{\sigma}-\frac{1}{\sigma^{3}} \cdot \sum_{j \neq i_{0}}\left(\Delta v_{j}\right)^{2}=0 .
$$

Multiplying both sides by $\sigma^{3}$, dividing both sides by $n-2$, and moving the term $\sigma^{2}$ to the other side, we conclude that

$$
\sigma^{2}=\frac{1}{n-2} \cdot \sum_{j \neq i_{0}}\left(\Delta v_{j}\right)^{2}
$$

Resulting probability distribution. Now, that we know the values of all the parameters, the probability distribution is uniquely determined: the probability $P_{i}$ that the actual transition is at location $i$ is proportional to

$$
L_{i} \sim \exp \left(-\frac{1}{2 \cdot \sigma^{2}} \cdot \sum_{j \neq i}\left(\Delta v_{j}\right)^{2}\right) .
$$

By using the above formula

$$
\sum_{j \neq i}\left(\Delta v_{j}\right)^{2}=\sum_{j=1}^{n-1}\left(\Delta v_{j}\right)^{2}-\left(\Delta v_{i}\right)^{2}
$$

and the fact that $\exp (a-b)=\exp (a) \cdot \exp (b)$, we conclude that

$$
\exp \left(-\frac{1}{2 \cdot \sigma^{2}} \cdot \sum_{j \neq i}\left(\Delta v_{j}\right)^{2}\right)=A \cdot S_{i}
$$

where we denote

$$
A \stackrel{\text { def }}{=} \exp \left(-\frac{1}{2 \cdot \sigma^{2}} \cdot \sum_{j=1}^{n-1}\left(\Delta v_{j}\right)^{2}\right),
$$

and

$$
S_{i} \stackrel{\text { def }}{=} \exp \left(\frac{\left(\Delta v_{i}\right)^{2}}{2 \cdot \sigma^{2}}\right)
$$

Thus, $P_{i} \sim S_{i}$, i.e.,

$$
P_{i}=c \cdot S_{i}
$$

for some constant $c$. This constant $c$ can be determined from the fact that the transition has to be at one of the locations $i$. Since the transition is only at one of the locations, this means that the probabilities $P_{j}$ of having transitions at different locations should add up to one: $\sum_{j=1}^{n-1} P_{j}=1$. Thus,

$$
1=\sum_{j=1}^{n-1} P_{j}=c \cdot \sum_{j=1}^{n-1} S_{j}=1
$$


hence

$$
c=\frac{1}{\sum_{j=1}^{n-1} S_{j}}
$$

and, finally, the probability $P_{i}=c \cdot S_{i}$ takes the form

$$
P_{i}=\frac{S_{i}}{\sum_{j=1}^{n-1} S_{j}}=\frac{\exp \left(\frac{\left(\Delta v_{i}\right)^{2}}{2 \cdot \sigma^{2}}\right)}{\sum_{j=1}^{n-1} \exp \left(\frac{\left(\Delta v_{j}\right)^{2}}{2 \cdot \sigma^{2}}\right)} .
$$

How to estimate accuracy: the idea. The mean square deviation $\sigma_{0}^{2}$ of the actual (unknown) transition depth from our estimate $i_{0}$ is, by definition, equal to

$$
\sigma_{0}^{2}=\sum_{i=1}^{n-1}\left(i-i_{0}\right)^{2} \cdot P_{i}
$$

Substituting the above expression for $P_{i}$ into this formula, we conclude that

$$
\sigma_{0}^{2}=\frac{\sum_{i=1}^{n-1}\left(i-i_{0}\right)^{2} \cdot \exp \left(\frac{\left(\Delta v_{i}\right)^{2}}{2 \cdot \sigma^{2}}\right)}{\sum_{j=1}^{n-1} \exp \left(\frac{\left(\Delta v_{j}\right)^{2}}{2 \cdot \sigma^{2}}\right)} .
$$

Resulting algorithm. First, we compute

$$
\sigma^{2}=\frac{1}{n_{2}} \cdot \sum_{j \neq i_{0}}\left(\Delta v_{j}\right)^{2},
$$

and then estimate $\sigma_{0}$ by using the above formula.

This algorithm leads to a reasonable result. We applied this algorithm to the seismic model of El Paso area derived in [1] (see also [11]). In this case, the transition is between the lower and the upper crust; see, e.g., [13].

For this map, we got $\sigma_{0} \approx 1.5 \mathrm{~km}$. This result is in good accordance with the fact that the difference between the border depth estimates coming from the seismic data and from the gravity data [4] is of the same order of magnitude (1-2 km).

\section{How to Fuse the Estimates of the TRANSition DePth AND How to Fuse The CORRESPONDING Models}

Available estimates for the transition depth. Now, we have two estimates for the transition depth:

- the estimate $i_{d}$ from the discrete (gravity) model, and

- the estimate $i_{0}$ from the continuous (seismic) model.

Accuracy of the available estimates for the transition depth. The estimate $i_{d}$ corresponding to the discrete model comes from a standard statistical analysis - as one of the parameters of the model. So, we can use the usual statistical techniques to estimate the standard deviation $\sigma_{d}$ of this estimate.

For the continuous estimate $i_{0}$, we already know how to compute its standard deviation $\sigma_{0}$.
How to fuse estimates of the transition depth: analysis of the problem. We would like to use the Maximum Likelihood Method to find the best fused estimate $i_{f}$ for the actual (unknown) transition depth $i$.

It is reasonable to assume that both differences $i_{d}-i$ and $i_{0}-i$ are normally distributed and independent. The probability densities corresponding to $i_{d}-i$ and to $i_{0}-i$ are therefore proportional to

$$
\exp \left(-\frac{\left(i_{d}-i\right)^{2}}{2 \cdot \sigma_{d}^{2}}\right) \text { and } \exp \left(-\frac{\left(i_{0}-i\right)^{2}}{2 \cdot \sigma_{0}^{2}}\right) .
$$

Since these uncertainties are independent, the likelihood of $i$ being the actual transition depth is proportional to the product

$$
\begin{gathered}
\exp \left(-\frac{\left(i_{d}-i_{f}\right)^{2}}{2 \cdot \sigma_{d}^{2}}\right) \cdot \exp \left(-\frac{\left(i_{0}-i_{f}\right)^{2}}{2 \cdot \sigma_{0}^{2}}\right)= \\
\quad \exp \left(-\left(\frac{\left(i_{d}-i\right)^{2}}{2 \cdot \sigma_{d}^{2}}+\frac{\left(i_{0}-i\right)^{2}}{2 \cdot \sigma_{0}^{2}}\right)\right) .
\end{gathered}
$$

Maximizing this likelihood expression is equivalent to minimizing the argument of the decreasing function $\exp (-z)$, i.e., minimizing the expression

$$
\frac{\left(i_{d}-i\right)^{2}}{2 \cdot \sigma_{d}^{2}}+\frac{\left(i_{0}-i\right)^{2}}{2 \cdot \sigma_{0}^{2}} .
$$

Differentiating this expression by $i$ and equating the derivative to 0 , we get the following result.

How to fuse estimates of the transition depth: resulting formula. Based on the estimates $i_{d}$ and $i_{0}$ for the transition depth, as the optimal estimate $i_{f}$ for the actual transition depth, we take the following value:

$$
i_{f}=\frac{i_{d} \cdot \sigma_{d}^{-2}+i_{0} \cdot \sigma_{0}^{-2}}{\sigma_{d}^{-2}+\sigma_{0}^{-2}} .
$$

Towards fusing actual maps. The fused value $i_{f}$ is our best estimate for the transition depth, i.e., for the border between the lower and upper zones which are, in our case, lower and upper crust.

In the discrete model:

- values corresponding to $i<i_{d}$ correspond to the upper zone, while

- values corresponding to the depths $i>i_{d}$ correspond to the lower zone.

Similarly, in the continuous model:

- values corresponding to $i<i_{0}$ correspond to the upper zone, while

- values corresponding to the depths $i>i_{0}$ correspond to the lower zone.

So, for depths $i \leq \min \left(i_{0}, i_{d}\right)$ and $i \geq \max \left(i_{0}, i_{d}\right)$, both models correctly describe the zone, and we can simply fuse the values from both models - e.g., similarly to how we fused the estimates for the transition depth.

For intermediate depths, we need to adjust the models, by replacing the values corresponding to the wrong zone by the 
nearest value from the correct zone. As a result, we get the following procedure.

\section{How to fuse the actual maps: general idea.}

- First, we adjust both models so that they both have a transition at depth $i_{f}$.

- Second, for each depth $i$, we merge the values $v_{i}^{\prime}$ and $v_{i}^{\prime \prime}$ corresponding to the adjusted models.

Let us describe this fusion in more detail.

Adjusting the discrete model. Adjusting the discrete model is (relatively) easy: we just replace the original depth $i_{d}$ with the new (more accurate) fused value $i_{f}$.

Adjusting the continuous model. When the more accurate transition depth $i_{f}$ is smaller than the transition depth $i_{0}$ corresponding to the continuous model $\left(i_{f}<i_{0}\right)$, this means that the values at depths $i$ between $i_{f}$ and $i_{0}$ are erroneously assigned to the the upper zone. In this case, the values $v_{i}$ for this $i$ must be replaced by the value of the nearest point at the lower zone, i.e., by the value $v_{i_{0}+1}$.

When the more accurate transition depth $i_{f}$ is larger than the transition depth $i_{0}$ corresponding to the continuous model $\left(i_{f}>i_{0}\right)$, this means that the values at depths $i$ between $i_{0}$ and $i_{f}$ are erroneously assigned to the the lower zone. In this case, the values $v_{i}$ for this $i$ must be replaced by the value of the nearest point at the upper zone, i.e., by the value $v_{i_{0}}$.

How to merge the adjusted models. For each depth $i$, we now have two adjusted values $v_{i}^{\prime}$ and $v_{i}^{\prime \prime}$ corresponding to two adjusted models. Let $\sigma^{\prime}$ and $\sigma^{\prime \prime}$ be the corresponding standard deviations. Then, similarly to what we have described earlier, we can compute the fused value $\widetilde{v}_{i}$ as follows:

$$
\widetilde{v}_{i}=\frac{v_{i}^{\prime} \cdot\left(\sigma^{\prime}\right)^{-2}+v_{i}^{\prime \prime} \cdot\left(\sigma^{\prime \prime}\right)^{-2}}{\left(\sigma^{\prime}\right)^{-2}+\left(\sigma^{\prime \prime}\right)^{-2}} .
$$

\section{ACKNOWLEDGMENT}

This work was supported in part by the National Science Foundation grants HRD-0734825 (Cyber-ShARE Center) and DUE-0926721, by Grant 1 T36 GM078000-01 from the National Institutes of Health, by Grant MSM 6198898701 from
MŠMT of Czech Republic, and by Grant 5015 "Application of fuzzy logic with operators in the knowledge based systems" from the Science and Technology Centre in Ukraine (STCU), funded by European Union.

The authors are thankful to Dr. Musa Hussein for his help and to the anonymous referees for their valuable suggestions.

\section{REFERENCES}

[1] M. G. Averill, A Lithospheric Investigation of the Southern Rio Grande Rift, University of Texas at El Paso, Department of Geological Sciences, PhD Dissertation, 2007.

[2] M. G. Averill, K. C. Miller, G. R. Keller, V. Kreinovich, R. Araiza, and S. A. Starks, "Using Expert Knowledge in Solving the Seismic Inverse Problem" International Journal of Approximate Reasoning, 2007, Vol. 45, No. 3, pp. 564-587.

[3] J. A. Hole, "Nonlinear High-Resolution Three-Dimensional Seismic Travel Time Tomography", Journal of Geophysical Research, 1992, Vol. 97, pp. 6553-6562.

[4] M. Hussein, private communication

[5] G. Klir and B. Yuan, Fuzzy Sets and Fuzzy Logic: Theory and Applications, Upper Saddle River, New Jersey: Prentice Hall, 1995.

[6] J. M. Lees and R. S. Crosson, "Tomographic inversion for threedimensional velocity structure at Mount St. Helens using earthquake data", Journal of Geophysical Research, 1989, Vol. 94, pp. 5716-5728.

[7] M. Maceira, S. R. Taylor, C. J. Ammon, X. Yang, and A. A. Velasco. "High-resolution Rayleigh wave slowness tomography of Central Asia", Journal of Geophysical Research, 2005, Vol. 110, Paper B06304.

[8] H. T. Nguyen and E. A. Walker, First Course on Fuzzy Logic, CRC Press, Boca Raton, Florida, 2006.

[9] O. Ochoa, A. A. Velasco, V. Kreinovich, and C. Servin, "Model fusion: a fast, practical alternative towards joint inversion of multiple datasets", Abstracts of the Annual Fall Meeting of the American Geophysical Union $A G U^{\prime} 08$, San Francisco, California, December 15-19, 2008.

[10] O. Ochoa, A. A. Velasco, C. Servin, and V. Kreinovich, "Model Fusion under Probabilistic and Interval Uncertainty, with Application to Earth Sciences", In: M. Beer, R. L. Muhanna, and R. L. Mullen (Eds.), Proceedings of the 4th International Workshop on Reliable Engineering Computing REC'2010, Singapore, March 3-5, 2010, pp. 81-100.

[11] C. Servin, M. G. Averill, N. Del Rio, L. Longpré, and V. Kreinovich, "Propagation and Provenance of Probabilistic and Interval Uncertainty in Cyberinfrastructure-Related Data Processing and Data Fusion", In: R. L. Muhanna and R. L. Mullen (eds.), Proceedings of the International Workshop on Reliable Engineering Computing REC'08, Savannah, Georgia, February 20-22, 2008, pp. 199-234.

[12] D. G. Sheskin, Handbook of Parametric and Nonparametric Statistical Procedures, Chapman \& Hall/CRC Press, Boca Raton, Florida, 2007.

[13] E. J. Tarbuck, F. K. Lutgens, and D. Tasa, Earth: An Introduction to Physical Geology, Prentice Hall, Upper Saddle River, New Jersey, 2010. 